\title{
Distribución, función y uso social de la tecnología desde la comunicación educativa. Un estudio de caso en el contexto cubano.
}

\begin{abstract}
(ㄷ)(1)(8) (2)
\end{abstract}
Distribution, show and I use social of the technology from the educational communication. A case study in the context Cuban.

Yulima D. Valdés Bencomo. ${ }^{1}$, Dagmar Santa Cruz Pérez. ${ }^{2}$, Victoria Ojalvo Mitrany. ${ }^{3}$ \& Luis Efraín Velastegui López. ${ }^{4}$

Recibido: 15-03-2021 / Revisado: 22-03-2021 /Aceptado: 12-04-2021/ Publicado: 05-05-2021

\begin{abstract}
.
DOI: https://doi.org/10.33262/concienciadigital.v4i2.1.1711

This case study addresses as a scientific problem from the socio-technical, the current limitations of educational and pedagogical communication as science and knowledge. The object of the case study focuses on the distribution, function and social use of technology to the understanding of the educational communication strategy, aimed at the work of sociocultural and community management in the Integral Transformation Workshop of the Neighborhood (TTIB-Canal) of the Cerro Municipality. The general objective is therefore: Explain the main factors that currently limit or hinder the distribution, function and social use of technology to the understanding of the educational communication strategy, aimed at sociocultural and community management work in the Workshop on Integral transformation of the Neighborhood (TTIB-Canal) of the Cerro Municipality, as specific: Make a socio-technical approach to the educational communication strategy directed to the work of sociocultural and community management in the TTIB-Canal of the Cerro Municipality. Determine the limitations that are presented in the educational communication strategy, aimed at sociocultural and

\footnotetext{
1 Universidad de la Habana, CEPES, La Habana, Cuba, yulima40@ gmail.com, Orcid: 0000-0003-4477-166x

${ }^{2}$ Universidad de la Habana, Rectorado, La Habana, Cuba, dagmar.santacruz@ rect.uh.cu, Orcid 0000-00026825-4555

${ }^{3}$ Universidad de la Habana, CEPES, La Habana, Cuba, victoria@ @epes.uh.cu, Orcid 0000-0003-0754-5663

${ }^{4}$ Ciencia Digital Editorial, Ecuador, luisefrainvelastegui@ cienciadigital.org
} 
community management work in the TTIB-Canal of the Cerro Municipality. Define the social impacts that are generated or not from the socio-technical point of view from the interaction of the strategy and describe its elements.

Sociocultural and community management has been defined, from a socio-technical approach as systems of strategic socio-technical processes through which the knowledge and socio-cultural and community practices of the community are legitimized as living heritage, they are assumed as identity value of the community and its transformative potentialities are channeled in negotiation contexts. It is considered that the current limitations of this cultural management are influenced by three types of determinants or factors, mainly the epistemic, technological and cultural-communicological factors.

Key words: educational communication, distribution, function and social use of technology.

\section{Resumen.}

El presente estudio de caso aborda como problema científico desde lo socio-técnico, las limitaciones actuales de la comunicación educativa y pedagógica como ciencia y conocimiento. El objeto de estudio de caso se centra en la distribución, función y uso social de la tecnología a la comprensión de la estrategia de comunicación educativa, dirigida al trabajo de gestión sociocultural y comunitaria en el Taller de transformación Integral del Barrio (TTIB-Canal) del Municipio Cerro. Se plantea entonces como objetivo general: Explicar los factores principales que limitan o dificultan en la actualidad la distribución, función y uso social de la tecnología a la comprensión de la estrategia de comunicación educativa, dirigida al trabajo de gestión sociocultural y comunitaria en el Taller de transformación Integral del Barrio (TTIB-Canal) del Municipio Cerro, como específicos: Hacer un abordaje socio-técnico de la estrategia de comunicación educativa dirigida al trabajo de gestión sociocultural y comunitaria en el TTIB-Canal del Municipio Cerro. Determinar las limitaciones que se presentan en la estrategia de comunicación educativa, dirigida al trabajo de gestión sociocultural y comunitaria en el TTIB-Canal del Municipio Cerro. Definir los impactos sociales que se generan o no desde lo socio-técnico a partir de la interacción de la estrategia y describir los elementos de la misma.

Se ha definido la gestión sociocultural y comunitaria, desde un abordaje socio-técnico como sistemas de procesos estratégicos socio-técnicos a través de los cuales los saberes y prácticas socioculturales y comunitarias de la comunidad se legitiman como patrimonio vivo, se asumen como valor identitario de la comunidad y sus potencialidades transformadoras se canalizan en contextos de negociación. Se considera que en las limitaciones actuales de esta gestión cultural inciden tres tipos de determinantes o factores principalmente el factor epistémico, tecnológico y el cultural-comunicológico.

Palabras claves: Comunicación educativa, distribución, función y uso social de la tecnología. 


\section{Introducción.}

Los primeros enfoques al surgir la preocupación por el estudio de los sistemas de información social tenían una visión más sociológica y estructural. Casi desde un principio se perfilaron dos tendencias como modo de entender esta dimensión macro social de los medios: una primera postura de carácter crítico fue inaugurada por la Escuela socio crítica de Frankfurt en la década de los treinta, la cual resalta las consecuencias negativas de los medios y trata de desmitificar su papel, por lo que se desmonta el poder y la racionalidad oculta en ellos y se definen las modificaciones desfavorables que tienen para las relaciones humanas. CEPES (1999). La segunda postura no considera la relación entre tecnología y poder, y puede caer en visiones infantilitas o inocentes de considerar a las tecnologías como entidades neutras. No obstante, en esta discusión más amplia y estructural existe otra tradición de línea de estudio que retoma el aspecto inmediato y fenomenológico que piensa la comunicación desde el hecho de la interacción. Dada la experiencia cotidiana popular que se construye a partir de relaciones cara-cara, lo comunicativo-popular por una parte retoma esta condición de la comunicación, lo que lleva a desarrollar diversas metodologías de trabajo grupal, pero no se queda en ellas, con la influencia de la pedagogía freiriana y la sociología materialista se materializa en lo político como esfera privilegiada de acción.

Se considera ante todo, según Álvarez de Zayas y Sampieri (1989) en sus reflexiones en común para que se consolide una metodología deben de existir tres elementos esenciales: El primero es la primera propiedad, un problema concreto a resolver y el segundo es el contraste o contradicción, o sea dos o más variables contrapuestas que como principio enmarca claramente al TTIB como espacio de intercambio y diálogo, a través de su proceso de gestión de trabajo sociocultural con su principio de contextualización, y se asume a la comunicación educativa para que el problema sea concreto. Por lo tanto, el trabajo sociocultural del Taller de trasformación Integral del Barrio (TTIB) supone que implica una comunicación.

Como tercera propiedad y última se considera la pertinencia social de la comunicación, lo que se está transformando a través de ella. La comunicación educativa es un proceso de interacción entre profesores, estudiantes y estos entre sí y de la escuela con la comunidad, que tiene como finalidad crear un clima psicológico favorable, para optimizar el intercambio y recreación de significados que contribuyan al desarrollo de la personalidad de los participantes".Ojalvo, V. (1992). Además es sistemática y transformadora. El presente estudio de caso nació en ámbitos diferentes a la gestión sociocultural y comunitaria de la comunidad en el TTIB a partir de la percepción de una trayectoria fallida de la comunicación desde la ausencia de una estrategia con enfoque socio-técnico, la falta de visión y sentido de pertenencia del gobierno local y la carencia de integración de los actores sociales relevantes.

A partir de este análisis se enmarcan las siguientes interrogantes: 
1. ¿Qué elementos socio-técnicos me ayudan a entender la estrategia de comunicación educativa, dirigida al trabajo de gestión sociocultural y comunitaria en el TTIB-Canal como una tecnología?

2. ¿Qué factores facilitan o dificultan la distribución, función y uso social de la tecnología, a la comprensión de la estrategia de comunicación educativa dirigida al trabajo de gestión sociocultural y comunitaria en el TTIB-Canal?

3. ¿Qué impactos sociales (económicos, políticos, culturales) se generan o no desde lo socio-técnico, a partir de la interacción de la estrategia de comunicación educativa dirigida al trabajo de gestión sociocultural y comunitaria en el TTIBCanal con la comunidad?

4. ¿Qué elementos describen la interacción de la estrategia de comunicación educativa dirigida al trabajo de gestión sociocultural y comunitaria en el TTIBCanal con los actores relevantes desde lo socio-técnico?

\section{Desarrollo}

\section{1-Enfoques de partidas y enfoques conceptuales. Contribución de los Estudios Sociales de la Ciencia y la Tecnología}

La tecnología es una necesidad del desarrollo civilizatorio humano. Su estudio es abordable de múltiples maneras ya que es parte de la cultura de los grupos humanos y contiene información de índole espiritual, material, intelectual, afectiva, de los modos de vida de una nación, y como la comprenden socialmente (sistema socioeconómico de producción, sistema de valores, derechos conquistados por el ser humano, tradiciones ceremonias y creencias) Este contenido distingue, cualifica y contextualiza el modo en que el ser humano crea y transforma su realidad produciendo nuevos sentidos en su existencia material y espiritual. En el orden estratégico en cuanto a la relación tecnologíasociedad se refiere a que hay valores, emergencias y cuestionamientos impostergables tales como: ¿A partir de que criterios de uso se diseña? ¿Quiénes están involucrados con la tecnología en cuestión? ¿Son reconocidos todos los involucrados en cuanto a participación? ¿Cómo les impacta a ellos y al objeto de aplicación? ¿A quiénes impacta como beneficio y a quienes como perjuicio y por qué? ¿Son pertinentes sus usos actuales para el contexto de aplicación? ¿Qué lecturas sociales ahí de ella a nivel de país, comunidad e individuos? ¿Cuál es la relación poder-tecnología-sociedad en este caso? ¿Qué mediaciones están presentes? Existen aportes de otros grupos de estudios como el herramental propositivo de los Estudios Sociales de la Ciencia y la Tecnología, capaz de reconocer complejidades de estas interrogantes y que nos ayudan a hacer este tejido reflexivo con las aportaciones tales como:

1. El enfoque de la construcción social de la tecnología de Wiebe Bijker y Trevor Pinch (2008) desde su flexibilidad interpretativa y los mecanismos de clausura según el constreñimiento del contexto social, tanto en las implicaciones para la tecnología, como para las comprensiones de las interrelaciones de actores sociales con esta. 
2. Las miradas sociales, políticas y éticas propias de la perspectiva de los Estudios Sociales de la Ciencia y la Tecnología Latinoamericanos relativas a la propuesta de las Tecnologías Sociales con sus preocupaciones por la comunidad, lo local, la participación, la inclusión social, la sustentabilidad y democracia económica en autores como Núñez(1998, 2008,2009), H. Dagnino,(2006, 2013) Martin Barbero(2007).

3. De modo solo puntual, la Teoría del Actor-Red de Michel Callon y Bruno Latour que entrelaza el factor tecnológico en relación con el entramado social, descubre varias formas de impacto de la tecnología, reconoce la agencia y el flujo de relaciones que se establece de lo físico, a lo político, lo tecnológico, lo semiótico y lo psicológico en especificidades irreductibles que reconocen la acción conjunta.

4. Desde la contribución a partir de los Estudios comunicológicos a la comprensión de la estrategia de comunicación educativa en función de la gestión del trabajo sociocultural y comunitario en el TTIB-Canal que será el estudio de caso

5. Desde la percepción de espacio en diálogo de construcción con el grupo de gestores del TTIB y los grupos humanos con patrimonio cultural y cognitivo en la comunidad

\section{1- Aportes necesarios desde la Construcción Social de la Tecnología. (COST)}

En sus conexiones, la tecnología resulta un importante elemento generador de políticas y estas generan, a su vez, tecnologías de implementación y otras normativas a partir de sus mutuos impactos económicos sociales (Bijker, 2005). Esto reviste una especial importancia para las comprensiones de democracia, respeto bioético y sostenibilidad en nuestros pueblos, sus comunidades e individuos. La tecnología es moldeada socialmente y las participaciones en los procesos de diseño, organización ejecución-uso, control, retroalimentación y rediseño del proceso tecnológico pueden definir la integralidad del mismo (Pinch, T.J. y Bijker, W.E.1995; Bijker, W.E. y Law J., 1992; Bijker, W.E.2005; Boszkowski, P. J., 2000)

El acercamiento teórico-metodológico del autor a este estudio desde la Construcción Social de Tecnología tuvo como objetivo seleccionar las miradas y aspectos que mejor develen las esencias socio-técnicas para la construcción de la función social de la tecnología a la comprensión de la estrategia. En su abordaje socio-técnico, el tejido de este concepto precisa de los "hilos conceptuales" que se seleccionó de la perspectiva que nos brinda Bijker y Pinch (1995), los artículos de Bijker (1995; 2005) y las entrevistas de Bijker (Boszkowski, P. J., 2000, Massare, s. a.).

Es importante señalar que, aunque no todos los matices de esta teoría se expresen de forma explícita en la formulación del concepto, estos condicionamientos están en su diseño de manera implícita (interactuada) y fueron necesarios para su reflexión y elaboración. En estas fuentes los autores critican y descartan los supuestos de imparcialidad como juicio de partida en ciencia, tecnología y sociedad, así como las llamadas "grandes causas generales" cómo únicas o "todo-determinantes“; con lo que este enfoque hace renuncias de la causalidad lineal. 
Este es un aspecto de valor para el campo socio-técnico que nos ocupa, ya que el manejo de expresiones culturales comporta aspectos multi-casuales.

La Construcción social de Tecnología entiende el establecimiento de relaciones tecnológicas y la tecnología como proceso social, no como ciencia aplicada aséptica y neutral; ya que en ella confluyen intereses muy diversos, beneficios y perjuicios bien diferenciados, por lo que se problematizan las interrelaciones sociales en torno al impacto de la tecnología. Se asumen estas posturas dentro del enfoque porque también privilegia los estudios de caso de la realidad y su mirada busca explicaciones sociales; prefiere estudios de casos que tengan " conexión clara con problemas sociales amplios", claras implicaciones políticas y normativas, encontrar problemas éticos, políticos, temas de etnicidad que develen los argumentos específicos de las negociaciones y las incongruencias fruto, muchas veces, de actitudes incoherentes y doble discurso". (Boszkowski, 2000:97-98).

Todo ello concuerda con el propósito de este análisis de profundizar en el contenido socio-técnico de la construcción de la función social de la tecnología a la comprensión de la estrategia. Este enfoque (COST) asume el interés en las lecturas de los mecanismos que desdoblan los actores para convencer a otros y "convencerse" a sí mismos de la validez de su discurso, lo cual se avino al empeño en revelar las posiciones conflictuadas y desacuerdos en el modo de manejar la función social de la tecnología a la comprensión de la estrategia entre grupos humanos que poseen motivaciones y percepciones diferentes en su manera de pensar y tratar la realidad sociocultural de su territorio.

La posición para la construcción de lo que se pretende se basa en el presupuesto bijkeriano, el cual asume la expresión que "la tecnología tiene que encajar en la sociedad" (Bijker en Massare, s.a.)Ninguna tecnología debe actuar a espaldas de los intereses, valores y motivaciones de los grupos sociales involucrados o impactados con su aplicación y este aspecto interviene en las apreciaciones de cómo debe aplicarse la tecnología de gestión cultural con respecto a los grupos portadores de los saberes y la comunidad. Una sociedad, como la nuestra que construye un proyecto social revolucionario debe tener como premisa "cambiar todo lo que deba ser cambiado" (Castro, 2000:5) también en lo socio-técnico.

\section{2- Aportes necesarios desde la Teoría del Actor-Red (TAR)}

En el caso de la Teoría del Actor-Red (TAR) los aportes que beneficiaron el abordaje que se necesita son muy puntuales en cuanto a comprender las interacciones humanas como negociaciones socio-técnicas (Latour, 2008) que establecen dinámicas heterogéneas donde los procesos técnicos y sociales se influyen y construyen mutua $\mathrm{y}$ sistemáticamente. Para el autor de este estudio o ensayo estas redes son socialmente heterogéneas debido a los diferentes actores humanos que despliegan otros recursos tecnológicos de apoyatura a la tecnología de gestión cultural que se analiza como: regulaciones, leyes, normativas. 
Se emplea el principio de escuchar a los actores y permitir que estos produzcan sentido desde sus propios mundos interpretativos. Michel Callon, desde su perspectiva del ActorRed, nos advierte que el dirigismo y la tecnocracia fracasan si no consideran el papel de la participación activa de los actores más heterogéneos en el sentido que lo asumimos en un determinado objetivo (Callon, M., 2008). Se valió también de legitimar la pericia de todos los actores que pueden aportar su saber y perspectiva en una comprensión más holística de los saberes y las problemáticas a su alrededor, apartándonos de la visión dicotómica de saber experto y no experto. Según Bruun y Hukinnen (2008:157) la TAR objeta la posibilidad de que la sociedad preceda a la acción, ya que es construida a través de ella.

Estos aportes de la Teoría del Actor-Red contribuyeron en este estudio a revelar debidamente el ensamble socio-técnico en que tiene lugar lo concerniente al mismo, a través de las trazas de los actores sociales relevantes que intervienen con las más diversas participaciones y con interacciones transversales que puedan develar. Esta perspectiva fue idónea para colaborar en la construcción social de la tecnología a la comprensión de la estrategia, en tanto nos muestra que "la Teoría del Actor-Red" expresa que para una acción exitosa se requiere alinear y coordinar de forma estable las identidades, los pensamientos y el comportamiento de otros actores o procesos. Por consiguiente, otorga una valiosa comprensión de los mecanismos de clausura y de construcción de los paradigmas tecnológicos y socio-técnicos. (Bruun y Hukinnen, 2008, p.158)

\section{3-Aportes necesarios desde la propuesta de las Tecnologías Sociales Latinoamericanas (TS)}

Las Tecnologías Sociales también son definidas como "productos, técnicas y o metodologías aplicables, desarrolladas en interacción con la comunidad y que representan soluciones efectivas de transformación social. " (Dagnino, 2006: 9). Otro autor vinculado al Tema, Caccia-Bava, toca explícitamente el asunto del poder y considera que las Tecnologías Sociales son mucho más que la mera capacidad de implementar soluciones para ciertos problemas, y que deben ser vistas como modos de empoderamiento social.

En sentido general las Tecnologías sociales son adecuaciones tecnológicas, propuestas de organización que viabilizan la democratización de las soluciones tecnológicas con lecturas de tecnología sobre la base de una práctica social con enfoque sistémico sustentable, que tiene en cuenta las necesidades, valores, creencias y la voluntad comunitaria, lo cual es de interés al propósito de conceptualizar la práctica tecnológica de la gestión del trabajo sociocultural y comunitario en el TTIB-Canal.

En esta mirada de las Tecnologías Sociales desde Latinoamérica se inclina por los aspectos locales, las posibilidades de desarrollo. Son un tipo de cambio tecnológico inclusivo/participativo social según las características contextuales de aplicación (Dagnino et al, 2006) pueden brindar al territorio y el interés por la interdisciplinariedad en el tratamiento de las dinámicas tecnológicas locales. 
Es, a su vez, una propuesta participativa de construcción de conocimiento, de hacer ciencia y tecnología para el desarrollo sustentable en todas sus potencialidades, respondiendo a las demandas por una mejor calidad de vida de las comunidades.

Para Cuba el tema del triángulo del poder, cultura, sociedad en los escenarios sociotécnicos supone ". La necesidad de repensar los desarrollos tecnológicos locales problematizando las dicotomías clásicas desarrollo/subdesarrollo, hegemonía/contra hegemonía“(Thomas, Fressoli, Lalouf, 2008: 80), Thomas, H. (2010), solo que en términos algo diferentes. "Las Tecnologías Sociales son métodos y técnicas que permiten impulsar procesos de empoderamiento de las representaciones colectivas de la ciudadanía, habitándolas para luchar los espacios públicos, las alternativas de desarrollo que se originan de las experiencias innovadoras y que se orienten a la defensa de los intereses de las mayorías" (Caccia-Bava, 2004:116)

Es interesante para nuestro estudio de caso específicamente la manera en que este enfoque latinoamericano significa el discurso anti hegemónico y legitima los discursos de los "terceros excluidos" generando mecanismos de inclusión social. Entre los aportes que consideramos oportunos e indispensables en cuanto a desarrollo local, desde el diálogo de saberes, están los argumentos más recientes sobre desarrollo local y participación, así como el papel y modo de impacto de los decisores locales en la trama social y en los emprendimientos según su modo cognitivo (Dagnino, 2013). La adecuación sociotécnica del emprendimiento, en consonancia con su contexto de utilización, podrá generar alternativas al desarrollo endógeno del territorio. En este sentido la estrategia de comunicación educativa, puede aportar al progreso social, y en su dinámica debe reflejarse la canalización de sus potencialidades transformadoras para la comunidad.

\subsection{Contribución a partir de los Estudios Comunicológicos}

Las distorsiones en la concepción de la realidad y la realidad subjetiva, sus posibilidades para penetrar los significados de las acciones sociales, la búsqueda de lo cualitativo hace que los paradigmas críticos, interpretativo de la comunicología sean de gran utilidad para el presente estudio de caso. Según Hilda Saladrigas (s.a.) y (2005), para estos paradigmas son de preferencia las agendas públicas, las rutinas profesionales, el análisis del comportamiento de los grupos e individuos y la interacción entre los individuos con su entorno. Estas vías de acceso cognitivo conectan con las características de nuestro estudio de caso, que opta por buscar las causas y factores condicionantes que limitan y sesgan la estrategia de comunicación educativa, y para ello necesita construir su dinámica desde lo socio-técnico.

El factor cultural-comunicológico comporta tres componentes o ámbitos interactuados en el orden individual: El horizonte referencial (Lo que ya sabe o cree que sabe, Mundo cognitivo) El horizonte de lectura (Lo que logra captar, percepción del individuo. Mundo computativo) El horizonte de sentido (Lo que logra interpretar, concebir a través de su cuerpo humanístico de conocimientos y su carga emotivo-experiencial. Mundo cogitativo). Portal, R (2003). 
Estos tres ámbitos del factor cultural-comunicológico actúan en un bucle recursivo, una especie de círculo genético de las capacidades intelectivas fruto de la organización individual congitante-computante. Este es el complejo tamiz que perfila el herramental de la percepción ético-humanista para la adjudicación de sentido a los eventos y que influye decisivamente las opiniones y la toma de decisiones. Estos presupuestos esclarecen los mecanismos mentales que tercian las decisiones que operan en nuestros decisores. Se aprecia en las instancias políticas un acercamiento indocumentado, totalmente subjetivo y terciado en la toma de decisiones, un acercamiento poco científico. En el ámbito local de la comunicación participativa no se utiliza un tono propagandístico o meramente divulgativo, sino el diálogo de comunicación para el desarrollo humano para el cambio social desde el diseño de los proyectos para lograr la interacción por apropiación comunitaria (Gumucio, 2001). Los espacios locales que generen proyectos socioculturales para el desarrollo humano y la transformación social necesitan este tipo de comunicación que:

- Coloca la toma de decisiones en mesa de negociación donde se consolida la capacidad de las comunidades de confrontar sus ideas sobre el desarrollo con el personal técnico y los planificadores.

- Refuerza el tejido social a través del fortalecimiento de las organizaciones propias a la comunidad. Protege la tradición y los valores culturales, al mismo tiempo que facilita la integración de nuevos elementos.

La comunicación y en particular las mediaciones a nivel local merecen espacios y profundizaciones, que no se justifican con este estudio de caso, pues nos interesa la repercusión de la estrategia de comunicación educativa en función de la gestión del trabajo sociocultural y comunitario en el TTIB-Canal para el desarrollo local, una de las riquezas del territorio que sería de interés para la cultura artística y el turismo, entre otras fuentes para financiar proyectos de desarrollo endógeno que regeneraran zonas menos favorecidas o deprimidas por diversas razones. Arellano, E. (2008): Portal, R (2003).Taller de Transformación Integral del Barrio "El Canal” (2001).

Por tanto, se estima que la estrategia de comunicación educativa en función de la gestión del trabajo sociocultural y comunitario en el TTIB-Canal, debe contemplar la posibilidad de que al potenciarse se ayude a la transformación sociocultural y socioeconómica del territorio, a través de su legitimación en el espacio socio-geográfico una vez que pueda establecer compromisos y cambiar actitudes. Taller de Transformación Integral del Barrio “El Canal” (2001).

$\mathrm{Si}$, se trata que el proceso de gestión se impregne de un enfoque integrador comunicológico en su interpretación, y que sensibilice a todos los actores sociales involucrados con esto, estaríamos en presencia de una comunicación educativa, pedagógica y participativa para alcanzar el empoderamiento comunitario. Este sería un importante paso para el desarrollo local. 


\section{5- Contribución desde la percepción de espacio en diálogo de construcción con el grupo de gestores del TTIB y los grupos humanos con patrimonio cultural y cognitivo en la comunidad}

A fin de construir un espacio de diálogo ajustado al propósito de construir la estrategia de comunicación educativa dirigida al desarrollo local y comunitario se concierta que este grupo de Estudios Sociales de la Ciencia y la Tecnología exigen que esta ideación contemple la estrategia como un proceso de secuenciación de acciones y a la gestión sociocultural y comunitaria como un sistema de procesos socio-técnicos, en tanto participan de manera interactuada en el campo cognitivo saberes generados y conservados vivos por la comunidad, el campo tecnológico en virtud de la aplicación de una tecnología para su manejo que debe ser socialmente consensuada en espacios de negociación y aplicada en co-construcción contextualizada según cuerdos mutuos con los diferentes grupos de la comunidad. Núñez, J., Montalvo L.F. y Figaredo, F. (2008).

Asimismo, estos aportes apuntan a que según la visión más progresiva de aplicación de tecnología interesada en atender el cambio para el desarrollo humano, la gestión sociocultural y comunitaria del patrimonio cultural y cognitivo, debe fomentar las potencialidades transformadoras de las prácticas culturales y canalizarlas a favor del bien de la comunidad y sus individuos, escuchando a los grupos sociales involucrados, alineando (rectificando, perfilando) la identificación con los saberes y la manera en que serán gestionados, los pareceres y mutua comprensión de los actores y los procesos.

Los aportes latinoamericanos en estos estudios señalan que se precisa tener en cuenta a todos los actores sociales en la red socio-técnica para el desarrollo comunitario con un estilo que propende a estándares locales más que nacionales y la contextualización de los requerimientos funcionales de la gestión sociocultural y comunitaria, por lo que se concilian propósitos, compromisos y actitudes para mover en sentido más equilibrado el posicionamiento asimétrico de los actores en cuanto a tecnología, poder y cultura desde las factibilidades en las cuotas locales de poder, saber, deseo y discurso. Núñez, J., Montalvo L.F. y Figaredo, F. (Comp.) (2008).

De estas consideraciones se unen los aportes de los Estudios Socioculturales para la búsqueda de la convergencia relacional socio-técnica en cuanto a la participación. Para ello se considera imprescindible operar sobre estas posiciones, satisfacciones, predisposiciones y posibilidades que se co-construyen, legitiman, alimentan e invaden recursivamente unas a las otras.

En este entramado de "patrones de interacción de tecnologías, instituciones, políticas, racionalidades y formas de constitución ideológica de los actores. “ (Thomas, 2008:199) se reconocen y legitiman los valores identitarios de los grupos humanos que se constituyen en su patrimonio. Estos necesitan legitimar su discurso y pareceres ya que son portadores de un "patrimonio vivo", dinámico, cambiante capaz de dialogar, discutir, rebatir, y estar de acuerdo o no con lo que acuerdan otros a nombre de ellos" (Guanche, 2008: 14) 
No considerar estos criterios anteriormente expuestos puede sufrir el riesgo tecnológico de la trasmutación artificial de las prácticas, la superficialidad de su comprensión y exposición, al rechazo y retraimiento de los grupos portadores naturales de las prácticas, a la pérdida del coeficiente humano del patrimonio y al enrarecimiento de las relaciones entre las instituciones culturales y los practicantes de la comunidad. Núñez, J., Montalvo L.F. y Figaredo, F. (Comp.) (2008).

\section{2-Distribución, función y uso social de la tecnología a la comprensión de la estrategia de comunicación educativa para el trabajo de gestión sociocultural y comunitaria en el TTIB-Canal como estudio de caso.}

Se hace necesario el análisis de la esencia de esta segunda parte del capítulo a partir de dos enfoques importantes: La dimensión sociocultural de la gestión de proyectos y la gestión de proyectos socioculturales. Veamos el primer enfoque: El mismo nos conduce a entender el carácter multifuncional de la gestión de proyectos el cual ayuda a comprender la diversidad de circunstancias en que esta resulta de utilidad, cuando se trata en primera instancia de asumir necesidades de cambios, desde una perspectiva estratégica e integradora.

La dimensión sociocultural de la gestión de proyectos asume, como área de interés, la pertinencia de prestar atención a la identificación de los modos de comportamiento humano y formas de organización social, que configuran rasgos de la cultura popular en el entorno en transformación, distinguiendo el papel de la participación en la construcción de identidades que promueven sentidos de pertenencia, y generan valores que pueden llegar a ser patrimoniales y constituyen la base que soporta toda obra de renovación necesaria.

Desde esta perspectiva puede apreciarse una intención de reconocimiento y una voluntad de compromiso que, aun cuando no advierte en lo sociocultural un sentido de totalidad transdisciplinaria, al menos repara en su inevitabilidad como condición caracterizadora de cualquier estrategia de cambio que apunte al desarrollo.

Veamos el segundo enfoque desde la gestión de proyectos socioculturales: El proyecto sociocultural emerge como opción para instrumentar el giro que la necesidad impone, centrando toda su atención en las implicaciones sociales y culturales de tal renovación. Desde este punto de vista el foco de atención se concentra en los seres humanos que hacen posible el logro de la finalidad de la propuesta, dando seguimiento al modo en que ésta fertiliza o inhibe el despliegue de sus potencialidades y poniendo énfasis en todos los recursos que pueden aportar a favor del cambio, sin escatimar saberes, experiencias, conocimientos científicos, filosóficos, religiosos o de cualquier índole.

El carácter complejo de los proyectos socioculturales hace posible la identificación en ellos de "dimensiones". Esas "dimensiones" resultan los componentes esenciales que definen a este tipo de proyecto de desarrollo y ninguna de ellas lo agota, por muy elevado que sea el modo en que se concretan. Precisamente en la articulación, complementariedad e integración de estas "dimensiones" radica la posibilidad de éxito en los proyectos 
socioculturales, ellas son: Dimensión política, comunicativa, económico-financiera, ambiental, educativa, investigativa, ética, estética, artística directiva y humanista.

Es importante apreciar que las "dimensiones" antes identificadas constituyen componentes de un sistema, considerando las estrechas y diversas relaciones y sinergias que pueden derivarse de su conjunción por lo que resulta importante reconocer que todas ellas, en su interacción dibujan un entramado complejo que, como totalidad, define a los proyectos socioculturales donde están contenidas.

Aceptar lo sociocultural como dimensión de la gestión de proyectos comprometidos con propósitos orientados hacia otras finalidades, o reconocer la existencia de proyectos socioculturales cuya dinámica interna se concreta a través de un sistema de dimensiones estrechamente interrelacionadas, no tiene que asumirse desde una perspectiva dicotómica. Ambas miradas constituyen extremos no excluyentes en el análisis de lo que he intentado que no se convierta en un simple juego de palabras.

\section{1-Factores que facilitan o dificultan la distribución, función y uso social de la tecnología, a la comprensión de la estrategia de comunicación educativa y su gestión sociocultural y comunitaria desde el TTIB y su entorno.}

Estos factores condicionantes deben comprenderse como un todo interactuado, que se presenta y actúa en la vida cotidiana de manera simultánea, y correlacionada, pero que en función de su mejor comprensión y explicación se ha separado en tres factores.

El factor epistémico: Involucra esencialmente el conocimiento superficial de los saberes y prácticas de la dinámica de la estrategia y su gestión sociocultural y comunitaria como objeto de estudio e investigación, el desconocimiento teórico de la propia gestión, así como la insuficiencia epistémica en su conceptualización desde el TTIB y la comunidad.

Esto trae consigo una importancia obvia, ya que para casi todos los que hemos tenido el privilegio de la vivencia irrepetible que constituye la gestión de proyectos socioculturales, resulta una verdad de Perogrullo el hecho de que la "ganancia principal" que se alcanza en tales experiencias son los aprendizajes derivados de los procesos de transformación social intencionalmente provocados.

Todo propósito educativo debe ser, ante todo, un ejercicio complejo de análisis y síntesis, que atienda a las esencias desde los más disimiles detalles, por lo que el reconocimiento de los elementos fundamentales para la formación humana constituye la base para la definición de lo que debe ser aprendido como prioridad, porque a partir de ello los seres humanos estarán en condiciones de decidir sus distintivas necesidades de aprender, y reconocer o construir sus propias estrategias para hacerlo.

EI factor tecnológico: En la localidad se evidencia la ausencia de percepción sistémica y de perspectiva estratégica a nivel local, por ende, es ineficaz su organización y ejecución. Asimismo, las deficiencias en el dominio de la gestión sociocultural y comunitaria, en general, traen consigo la falta de actitud tecnológica en los gestores 
culturales de la localidad desde la importancia del uso del conocimiento que necesitamos y la distribución del conocimiento que necesitamos (escuelas, la prensa, instituciones)

El factor cultural-comunicológicos: Desde lo cultural-comunicológicos se aprecia la pérdida del sentido comunicológico de la difusión de la gestión sociocultural y comunitaria.

Asimismo, se advierte la pérdida de sensibilidad ante la otredad/mismidad de la condición humana, evidente en la oposición al diálogo, imposición de discursos hegemónicos y negación de la manifestación sociocultural de grupos humanos como expresiones identitarias en el Municipio El Cerro por los decisores políticos y esta situación crea incoherencia de discurso a nivel comunitario. Todo ello incapacita la apreciación y canalización de las potencialidades de la gestión sociocultural y comunitaria para la transformación social y el desarrollo local.

Al considerar en todo este engranaje a la comunicación como proceso, función, herramienta necesaria para el cambio y como eje transversal de todo el proceso, no podemos aceptar el desarrollo de acciones comunicativas aisladas a pesar de las buenas intenciones de personas o grupos implicados en la propuesta, necesariamente estamos obligados a pensarlas en sus interconexiones desde y hacia donde vayan o retornen. Comprender el significado de las comunicaciones para el fortalecimiento de los rasgos identitarios desde el proyecto sociocultural que lo diferencian de otros y fortalecen su imagen en los públicos vinculados (directa o indirectamente) al cumplimiento de los objetivos propuestos, es una condición indispensable para su adecuada gestión; lo cual exige de toda una actitud proactiva en relación con el proyecto en sí mismo y con las comunicaciones en particular.

2.2-Impactos sociales (económicos, políticos, culturales) que se generan o no desde lo socio-técnico, a partir de la interacción de la estrategia de comunicación educativa y su gestión sociocultural y comunitaria desde el TTIB y su entorno.

Conocimiento-ciencia-tecnología e innovación: Están condicionados por factores económicos, políticos culturales y sociales. La totalidad Social sobre los PSCT: ¿Qué impactos sociales se están generando o no desde lo socio-técnico? Todo esto son Procesos sociales que están condicionados por la sociedad: Procesos políticos: Impactan a la sociedad que los acogen, Relaciones de doble tráfico Agentes políticos de la sociedad: Empresa, Estado:

1. Proceso social

2. Grupos sociales relevantes: El Estado el cual distribuye el presupuesto público de la Ciencia, la tecnología y la innovación (CTI)

3. Dimensión de las políticas: ejemplo: Cuba tiene que construir una política de CTI y esto condiciona a lo local y lo comunitario.

Desde la Función social del conocimiento: Es importante usar el conocimiento que necesitamos y distribuir el conocimiento que necesitamos (escuelas, la prensa, instituciones) Si una sociedad pierde a los jóvenes que tiene que formar, entonces ahí hay 
que rectificar algo, y desde nuestro entorno existen muchos jóvenes que andan rondando por los circuitos del conocimiento, muchos de ellos se han podido rescatar incorporándolos a proyectos socioculturales y comunitarios con fundamento sociotécnico. El conocimiento se jubila, se incorpora, o se pierde cuando no es usado.

Tiene que ser movible y usado en la sociedad (Formación universitaria en todos sus matices y capacitación de factores) La reflexión social de la Tecnología que nos lleva a este estudio de caso, es que una sociedad no puede vivir sin eso. No opera sin conocimiento, ya que somos seres socio-técnicos que estamos moldeados por la tecnología es lo que nos permite vivir en este mundo contemporáneo, la tecnología está de manera inmediata y moldea en la vida de los seres humanos. Es parte de nuestras soluciones, pero es parte de nuestros problemas.

¿Será posible construir un desarrollo sostenible basado en el conocimiento? Esto se hace imposible con tan solo citar el ejemplo de este estudio de caso donde la comunicación transverzaliza todo el proceso de la vida cotidiana.

\section{Elementos que describen la interacción de la estrategia de comunicación educativa y su gestión sociocultural y comunitaria con los actores relevantes desde lo socio- técnico.}

No es suficiente el diseño de las comunicaciones en la gestión sociocultural a través de los proyectos descritos en planes de acción o campañas. Es determinante para ello, una visión socio-técnica que reconozca la medida en que las comunicaciones van a incidir en los procesos de cambio y cómo van a contribuir en la integración de los actores sociales relevantes a esas estrategias a través del diálogo.

Se refiere a cómo las comunicaciones ayudan a todos a comprender su papel y lugar en esa transformación, cómo grupo gestor y actores sociales relevantes se integran en la delineación de su futuro y del propio proyecto.

Los diseños comunicacionales han de permitir el movimiento de las comunicaciones (ascendente, descendente, horizontal y transversal) en toda la estructura interna del proyecto sociocultural y de éste con los diferentes actores sociales relevantes (promotores naturales, líderes comunitarios "formales o informales" representantes de instituciones de apoyo, gobiernos locales, grupos beneficiarios, financistas, entre otros) que se mueven en el entorno de proyecto y se vinculan a él desde las propias actividades.

Actores sociales relevantes (ASR) (Este término proviene de la Sociología de la Tecnología) Grupos sociales relevantes que están asociados a esas acciones o Red de actores que dan forma a esas decisiones. (Pueden ser positivos o negativos) Se deben analizar las fortalezas y las debilidades. Gobierno Municipal (CAM-Cerro), Federación de Mujeres cubanas (FMC) Cultura Municipal, Consejos populares con sus delegados

Selección de la ruta tecnológica: Es importante caminar en la medida que los grupos sociales lo hagan posible, tener en cuenta la trayectoria socio- tecnológica del fenómeno 
en cuanto a: Sentido en el tiempo, Movimiento en el tiempo y Transformación en el tiempo.

La tecnología se gesta con todos los recursos humanos y materiales que hacen faltan, donde los actores sociales relevantes (ASR) como condición indispensable deben de estar alineados, es por eso que muchas tecnologías se vuelven truncas, porque se tronchan o se frenan en el tiempo. Es de aquí donde parte la idea de las trayectorias tecnológicas y su languidez, y la pregunta que nos hacemos al respecto: ¿Qué trayectorias tecnológicas voy a desenvolver y proyectar?

La ciencia y la tecnología son construcciones sociales, entiéndase, la que tenemos y la que no tenemos, por lo tanto, de acuerdo a este estudio de caso la trayectoria tecnológica de la estrategia debe responder a las necesidades sentidas en los espacios de diálogo de los grupos como seres socio-técnicos enfocados en la búsqueda de soluciones reales y efectivas.

"Ciencia-en el sentido muy amplio que le he atribuido- y cultura, unidos, nos ayudan a entendernos a nosotros mismos como Nación, como grupos, como individuos; nos ayudan a tomar decisiones personales y colectivas"

Jorge Núñez, 2001

“La tecnología tiene que encajar en la sociedad...donde debe haber una conexión con la cultura y las creencias de una sociedad"

Wiebe Bijker, 2005

“Las relaciones puramente sociales sólo pueden ser encontradas en la imaginación de los sociólogos, relaciones puramente técnicas sólo se encuentran en el terreno de la ciencia-ficción. Lo técnico es socialmente construido y lo social es tecnológicamente construido. Todos los ensambles estables son estructurados al mismo tiempo tanto por lo técnico como por lo social**

Wiebe Bijker, 2005

\section{Metodologia.}

Se emplearon diversos métodos de la investigación social, que permiten la reflexión epistemológica y sistemática acerca de los métodos y procedimientos para la búsqueda de nuevos conocimientos sobre la realidad social en la comunidad.

Se asume el paradigma socio crítico y la metodología de la investigación-acción, en la búsqueda intencionada de investigar la distribución, función y uso social de la tecnología desde la comunicación educativa sustentada en la reflexión y la acción.

- El método Histórico-lógico se aplicó para conocer la evolución histórica de los procesos y conceptos analizados y su articulación en la lógica de las ciencias, como son los de extensión universitaria, gestión sociocultural, construcción social de la 
tecnología, actor red, enfoque socio técnico, tecnologías sociales y estrategias comunicativas.

- El método Analítico-Sintético: Se utilizó para la caracterización de la distribución, función y uso social de la tecnología.

\section{Metodología de investigación}

Para dar cumplimiento a los objetivos propuestos, se desarrolló una investigación mixta compleja: Hernández Sampieri, Fernández, y Baptista (2006):

"Los enfoques mixtos complejos representan el más alto grado de integración o combinación entre los enfoques cualitativo y cuantitativo. [...] En los diseños mixtos complejos, ambos enfoques se entremezclan o combinan en todo el proceso de investigación, o al menos, en la mayoría de sus etapas. [...] Algunas de las características de estos diseños son: Se recolectan simultáneamente datos cualitativos y cuantitativos, a varios niveles o diferentes secuencias [...] se puede desarrollar teoría emergente. (Hernández Sampieri, Fernández, y Baptista, p.784)

Se refleja, además lo correspondiente a los estudios de casos estos según autores, se definen como:

"Estudios que al utilizar los procesos de investigación cuantitativa, cualitativa o mixta; analizan profundamente una unidad para responder al planteamiento del problema, probar hipótesis y desarrollar alguna teoría“. (HernándezSampieri, Fernández, y Baptista, p.224)

\section{Resultados}

1. Dada la necesidad de aportar respuestas socio-técnicas a los problemas de la gestión sociocultural y comunitaria y que este estudio de caso responda a la política del país trazada en los Lineamientos de la Política Económica y Social del Partido y la Revolución, prestamos especial atención a los perfiles de los lineamientos $137,138(67)$ y 139 concernientes al futuro que deseamos construir en materia de Ciencia, Tecnología, y respondemos a los deseos del pueblo(68) de participar activamente con soluciones tecnológicas en este empeño, plasmados en el lineamiento.

2. Asimismo, atendemos especialmente al lineamiento 163, en el ámbito de la Cultura, sobre "la defensa de la identidad y la conservación del patrimonio cultural, el enriquecimiento de la vida cultural de la población y el potenciar del trabajo comunitario (69), y en este ánimo se recomienda: Consolidar estrategias con presupuestos comunicológicos, socios técnicos, educativos y pedagógicos en el trabajo de interacción de la gestión sociocultural y comunitaria de la población con inclusión social desde los TTIB. 


\section{Conclusiones.}

Este estudio de caso reveló su identificación con la coyuntura histórica que transita nuestro país, atendiendo a los lineamientos de la Política Económica y Social del Partido y la Revolución y los Objetivos de trabajo de la Conferencia del PCC, en lo concerniente a lo trazado y lo ampliado por las intervenciones públicas tanto en Ciencia, Tecnología e Innovación y como en el ámbito de la cultura.

Se ha hecho converger en este estudio de caso de la estrategia de comunicación educativa dirigida al trabajo de gestión sociocultural los Estudios Sociales de la Ciencia y la Tecnología y la Innovación y sus aportes desde los enfoques de construcción social de Tecnología, la propuesta latinoamericana de las Tecnologías sociales, la teoría del Red de Actor y la contribución desde la percepción de espacio en diálogo de construcción. También se utilizaron las contribuciones de los Estudios comunicológicos Dando respuestas a los objetivos planteados en este estudio de caso:

- Los presupuestos teóricos con que se triangulo de modo múltiple el enfoque de la estrategia y su gestión sociocultural y comunitaria, permitió definirla desde un abordaje socio-técnico, como sistema de procesos estratégicos socio-técnicos a través de los cuales los saberes y prácticas socioculturales y comunitarias de la comunidad se legitiman como patrimonio vivo.

- El abordaje socio-técnico permitió interpretar a la estrategia y su gestión sociocultural y comunitaria desde las Tecnologías sociales en tanto debe de ejecutársele con enfoque de inclusión social en todos y cada uno de los procesos de gestión, con diálogos horizontales y anti hegemónicos.

- Asimismo, el enfoque construido de la estrategia y su gestión sociocultural y comunitaria confirmo la idea a defender y explicar el efecto esperado de los tres factores de signo negativo que limitan la dinámica socio-técnica de la estrategia

\section{Referencias bibliográficas.}

Álvarez de Zayas, C. ,Sampieri. H (1989). Fundamentos teóricos de la dirección del proceso docente educativo en la superior cubana. Ciudad de la Habana.

Arellano, E. (2008): La estrategia de comunicación orientada al desarrollo de la cultura organizacional. Razón y Palabra, vol. 13, núm. 2, mayo-junio. Instituto Tecnológico y de Estudios Superiores de Monterrey, Estado de México, México

Barbero, M. (2007). Comunicación y Cultura: Unas relaciones complejas. En R. Portal y M. Recio, (comp.) Comunicación y comunidad (pp.25-34) La Habana:

Bijker, W.E. (1995). Of Bicycles, Bakelites, and Bulds. Toward a Theory of Sociotechnical Change. (De bicicletas, bakelita y bombillas. Hacia una teoría del cambio socio-técnico) Cambridge, (Mass.), The MIT Prees.

Bijker, W.E. (2005). ¿Cómo y porque es importante la tecnología? En Redes, mayo, vol.11, no. 021, pp.19-53. 
Bijker, W.E y Pinch, T. (2008).Actos, actores y artefactos. Sociología de la Tecnología (pp.19-62), Publisher: Buenos Aires: Bernal: Universidad Nacional de Quilmea. Editors. H Thomas, A. Buch.

Bijker, W.E y Law, J. (1992). Shaping Technology/Building Society. Studies in Sociotechnical Change, (Dar forma a la Tecnología y construir la sociedad. Estudios sobre el cambio tecnológico) Cambridge, (Mass.), The MIT Prees

Boszkowski, P. J. (2000). Del laboratorio a la ciudad: Wiebe Bijker habla de la evolución de los Estudios Sociales de la Tecnología. En Redes, vol.7, no.016, diciembre, pp.89-106

Bruun y Hukinnen, J. (s. a) (2008.:157) Cruzando Fronteras: Un diálogo entre tres formas de comprender el cambio tecnológico, en Actos, actores y artefactos. Herramientas para el análisis de los procesos de cambio tecnológico y cambio social, s.e. pp. 140-168.

Caccia-Bava, S. (2004). Tecnología social y desenvolvimiento local. En R. Dagnino, F. Cruvinel, H. Tahan, A. E. Lassance Jr., J. S. Pedreira, J. de Oliveira, Tecnología social: uma estratègia para o desenvolvimiento (Tecnología social y desarrollo social. En Tecnología social una estrategia para el desarrollo) (pp.103-116) Río de Janeiro: Fundación Banco do Brasil.

Callon M. (2008). La dinámica de las redes tecno-económicas. Publicado originalmente como "The Dynamics of Techno-economic Networks". En Coombs, R.; P. Saviotti y V. Walsh (eds.) (1992), Technological Change and Company Strategies. Londres. Harcourt Brace Jovanovich Publishers.

Castro, F. (2000, mayo 2) Discurso del Comandante en Jefe Fidel Castro Ruz en la celebración del Día de los Trabajadores el Primero de mayo. Granma, pp.4-5.

CEPES. (1999). Centro de estudios para el perfeccionamiento de la Educación Superior. Universidad de la Habana. Revista Cubana de Educación Superior. Editorial UH

Dagnino, R. (2013, febrero) "Política Científica y Tecnológica y Tecnologías Sociales" Conferencias impartidas en Ciclo de Conferencias sobre Política Científica y Tecnológica y Tecnologías Sociales, realizando en Hotel Habana Paseo, La Habana, Cuba.

Dagnino, R.; Bagattolli, C.; Tahan, H.; Fraga, L.; Tait, M. M., Pavan, M.et al. (2006) Primer Fórum de Redes de Tecnologías Sociales. Cuaderno de textos base para las discusiones. Una nueva cultura de participación para el desarrollo sustentable. Grupo de análisis de Políticas Públicas (GAPI) del Departamento de Política Científica y Tecnológica (DPCT) de la Universidad Estadual de Campinas (Unicamp) 
Guanche, J. (2008). El imaginado "Patrimonio Inmaterial” ¿Un acercamiento a la verdad o una falsedad engañosa? En revista Perfiles de la Cultura Cubana, enero-abril, p. 1-15. La Habana.

Gumucio, A. (2001). Haciendo Olas: Comunicación participativa para el cambio social. The Rockefeller Foundation. New York, pp.5-41.

Hernández-Sampieri, R., Fernández, C. y Baptista, P. (2006). Metodología de la Investigación, Cuarta edición. Mc. Graw Hill Interamericana.

Latour, B. (2008). Reensamblar lo social: una introducción a la teoría del actor-red. Buenos Aires: Ediciones Manantial.

Massare, B. (s.a) Wiebe Bijker. La Tecnología tiene que encajar en la sociedad. Buenos Aires:(s.e)

Núñez, J. (2001). Ciencia y Cultura: medio siglo después. En ciencia, Tecnología, Sociedad y Cultura en el cambio de siglo, Biblioteca Nueva, OEL, López Cerezo, J. A y Sánchez Ron (eds), 2001, Madrid, pp. 89-109.

----- (2008) Indicadores y relevancia social del conocimiento. En Universalización y cultura científica para el desarrollo local, coord. Irene Trelles y Miriam Rodríguez, Editorial Félix Varela, La Habana, pp.77-102.

----- (2009) La cuestión de la democratización de la ciencia como asunto epistemológico, ético y político. Disponible en Revista Digital Universidad y Sociedad vol.1, no.1. Universidad de Cienfuegos Carlos Rafael Rodríguez, en www.ecf.edu.cu

Núñez, J., Montalvo L.F. y Figaredo, F. (Comp.) (2008). Pensar Ciencia, Tecnología y Sociedad. La Habana: Editorial Félix Varela.

Ojalvo, V. (1992). La Comunicación. Impresiones Ligeras. Ciudad de La Habana.

Portal, R (2003). Un estudio de prácticas comunicativas de los Talleres de transformación de Integral del barrio en Ciudad de la Habana. Tesis en opción al grado científico de Doctora en Ciencias de la Comunicación. Facultad de Comunicación de la Universidad de La Habana

Saladrigas, H. (s.a.) La epistemología de la comunicación, terreno de urgente reflexiones y necesarias aportaciones. Introducción al libro Comunicología. Temas actuales, Facultad de Comunicación. La Habana: Universidad de la Habana.

---- (2005) Coordenadas cubanas para un fenómeno: Fundamentos para un enfoque teórico-metodológico de la investigación de la Comunicación Organizacional. Tesis en opción al grado científico de Doctora en Ciencias de la Comunicación Social. Facultad de Comunicación de la Universidad de La Habana 
Taller de Transformación Integral del Barrio "El Canal" (2001). Proyecto Comunitario Bongo Itá. Municipio Cerro: Lic. Esperanza Cantillo Mestre.

Thomas, H. (2010). De las tecnologías apropiadas a las tecnologías sociales. Conceptos/estrategias/diseños/acciones. Grupo de Estudios sociales de la Tecnología y la Innovación IEC/UNQ.ONICET. Disponible en http://inti.gob.ar./bicentenario/documentoslibro/pdf/anexo 4/jornadas tecno soc hernan th omas. pdf

Thomas, H. (2010). Estudios Sociales de la Tecnología en América Latina en İCONOS 37, pp.35-53

Thomas, H., Fressoli, M.; Lalouf, A. (2008). Actos, actores y artefactos. Herramientas para el análisis de los procesos de cambio tecnológico y cambio social. (s.e)

【| Ciencia 


\section{PARA CITAR EL ARTÍCULO INDEXADO.}

Valdés Bencomo, Y. D., Cruz Pérez, D. S., Ojalvo Mitrany, V., \& Velastegui López, L. E. (2021). Distribución, función y uso social de la tecnología desde la comunicación educativa. Un estudio de caso en el contexto cubano. ConcienciaDigital, 4(2.1), 92-112. https://doi.org/10.33262/concienciadigital.v4i2.1.1711

\section{Liencia}

El artículo que se publica es de exclusiva responsabilidad de los autores y no necesariamente reflejan el pensamiento de la Revista Conciencia Digital.

El artículo queda en propiedad de la revista y, por tanto, su publicación parcial y/o total en otro medio tiene que ser autorizado por el director de la Revista Conciencia Digital.

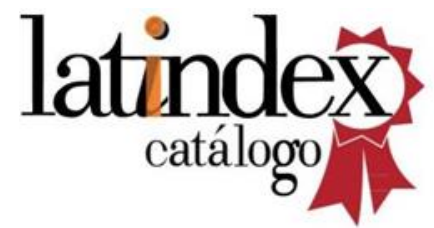

